\title{
Tracking Control of Chaotic Systems via Optimized Active Disturbance Rejection Control
}

\author{
Fayiz Abu Khadra $\mathbb{D}$ \\ Mechanical Engineering Department, King Abdulaziz University, Rabigh, Saudi Arabia \\ Correspondence should be addressed to Fayiz Abu Khadra; fabukhadra@kau.edu.sa
}

Received 22 April 2018; Revised 28 July 2018; Accepted 4 September 2018; Published 19 September 2018

Academic Editor: Anna Vila

Copyright (C) 2018 Fayiz Abu Khadra. This is an open access article distributed under the Creative Commons Attribution License, which permits unrestricted use, distribution, and reproduction in any medium, provided the original work is properly cited.

\begin{abstract}
For tracking control of chaotic systems, we develop an active disturbance rejection (ADR) control method. Using the first state of the system as the only available state, a time-varying bandwidth extended state observer reconstructs the remaining states and the total disturbance. A time-varying bandwidth feedback controller forces all the states of the system to follow exactly the reference signal and its derivative. The parameters of the ADR controller are optimized using a genetic algorithm. As the objective function, we chose the weighted sum of the integral of the absolute error and the integral of the absolute control signal. Two chaotic systems-the Duffing system and the Genesio-Tesi system-are considered in computer simulation tests. Results of these simulations are presented to demonstrate the effectiveness of the ADRC method in controlling chaotic systems.
\end{abstract}

\section{Introduction}

Chaos is the complex, unpredictable, and irregular behavior of systems. The behavior is very sensitive to a slight change in the initial conditions. Chaotic systems are very interesting nonlinear systems and have been intensively studied in numerous fields. Indeed, chaos can be found in many engineering systems such as oscillators, chemical reactions, robotics, lasers, and secure communications. Controlling a chaotic system requires designing a controller that stabilizes the system towards equilibrium points or forces the system to follow a time-varying reference signal. Transforming the system's response into useful signals is important in practice. Towards this end, a variety of control methods have been proposed, ever since the pioneering work reported in [1]. Examples include sliding mode control [2-8], adaptive control [9-18], and backstepping control [19, 20].

The control methods cited above are examples from two categories. The first assumes that, for a system model described accurately by a mathematical model, all states are available, neglecting uncertainties and external disturbances. This leads to a simple control method. The second assumes that the mathematical description of the system is partially known. This leads to a complex control method. In most cases in practice, only one state is available; the parameters of the system are uncertain and the system is subject to external disturbances. These uncertainties and external disturbances affect the performance of the controller. Therefore, it is essential to develop techniques that provide reliable estimates of the other states and the external disturbances, from the available single output. This problem can be solved if an observer is used. The observer design is a branch of control theory, for which solutions exist for linear and nonlinear systems. An observer is designed to reconstruct the unmeasured states and the external disturbance.

The active disturbance rejection (ADR) control method [21-23] is a nonlinear control method that is efficient and easy to implement. In addition, it is an effective method in dealing with uncertain nonlinear systems subject to external disturbances. In the (ADR) control method the unknown dynamics of the system and the external disturbances are considered the "total disturbance". The extended state observer (ESO) reconstructs the missing states and the total disturbance. These outputs of the ESO are used by a feedback controller. The procedure keeps the controller relatively simple, as the plant is reduced to a chain of integrators. 
The objective of this paper is as follows:

(i) To control chaotic systems with uncertain parameters, in the presence of external disturbances using ADRC method.

(ii) To optimize the ADRC controller parameters so that the system performs well by rejecting the total disturbances acting on the system and minimizing the tracking error to improve the overall system performance.

(iii) To suppress the peaking phenomena and improve the controller performance by using the time-varying observer and controller bandwidths.

As an objective function, a weighted sum of the integral of the absolute error and the integral of the absolute control signal is used, so that good tracking and concomitantly a smaller control signal can be produced. Two well-known examples are considered: the Duffing system (DS) as a typical secondorder chaotic system and the Genesio-Tesi system (GTS) as a typical third-order chaotic system.

The organization of this article is as follows. Section 2 states the problem at hand in mathematical terms. Section 3 describes the ADRC method. In Section 4, simulation experiments are performed to show the effectiveness of the proposed scheme in controlling the Duffing and Genesio-Tesi chaotic systems. Section 5 concludes the paper.

\section{Problem Formulation}

In this study, the chaotic systems to be controlled are a class of $\mathrm{n}$-th order single-input and single-output (SISO) continuous nonlinear systems, described by the dynamic equation:

$$
\begin{gathered}
\dot{x}_{1}=x_{2} \\
\dot{x}_{2}=x_{3} \\
\vdots \\
\dot{x}_{n}=f(X, t)+\Delta f(X, t)+d(t)+u(t) \\
y=x_{1}(t)
\end{gathered}
$$

where $X=\left[x, \dot{x}, \ldots, x^{n-1}\right]^{T}=\left[x_{1}, x_{2}, \ldots, x_{n}\right]^{T} \in \mathbb{R}^{n}$ is the state vector, $f(X, t)$ is function of the states and time, not exactly known but assumed to be continuous and bounded, $y \in R$ is the output, and $u(t) \in R$ is the control signal. The functions $\Delta f(X, t), d(t)$ are the bounded uncertain term and the disturbance term, respectively. In mathematical form,

$$
\begin{array}{r}
|\Delta f(X, t)| \leq \alpha, \\
|d(t)| \leq \beta
\end{array}
$$

where $\alpha$ and $\beta$ are positive constants. The given reference vector $R(\mathrm{t})$ is assumed to be bounded and has up to $(n-1)$ bounded derivatives; that is,

$$
R(t)=\left[r, \dot{r}, \ldots, r^{(n-1)}\right]^{T}=\left[r_{1}, r_{2}, \ldots, r_{n}\right]^{T}
$$

The aim of a control problem is to design a controller $u(t)$ to force the system output $y(t)$ to follow the given bounded reference signal $R(t)$ and its derivatives, that is, to minimize the tracking error defined as $E(t)=X(t)-R(t)$,

$$
\lim _{t \rightarrow+\infty}\|E(t)\|=\|X(t)-R(t)\| \longrightarrow 0
$$

where $\|\cdot\|$ is the Euclidian norm (or 2-norm) of a vector.

\section{ADR Controller}

In practice, only partial information of the mathematical model is known for most control systems; that is, the system has uncertainties and external disturbance. The main part of ADR controller is the ESO [18]. The main idea of the ESO is to use an additional state $x_{n+1}=f(X, t)+\Delta f(X, t)+d(t)$ so that system (1) can be written in state equation form as

$$
\begin{gathered}
\dot{x}_{1}=x_{2} \\
\dot{x}_{2}=x_{3} \\
\vdots \\
\dot{x}_{n}=x_{n+1}+u(t) \\
\dot{x}_{n+1}=\dot{F}(X, t) \\
y=x_{1}(t)
\end{gathered}
$$

Here $x_{n+1}=F(X, t)$ is the total disturbance or the extended state of the system. Note that the extended state can be considered as total unknown factor including linear/nonlinear function of state and the disturbance part.

The ESO in its nonlinear version takes the form:

$$
\begin{gathered}
\dot{\hat{x}}_{1}=\widehat{x}_{2}+\alpha_{1} \omega_{o}(t)\left(y-\widehat{x}_{1}\right) \\
\dot{\hat{x}}_{2}=\widehat{x}_{3}+\alpha_{2} \omega_{o}^{2}(t)\left(y-\widehat{x}_{1}\right) \\
\vdots \\
\dot{\hat{x}}_{n}=\widehat{x}_{n+1}+\alpha_{n} \omega_{o}^{n}(t)\left(y-\widehat{x}_{1}\right)+u(t) \\
\dot{\hat{x}}_{n+1}=\alpha_{n+1} \omega_{o}^{n+1}\left(y-\widehat{x}_{1}\right)
\end{gathered}
$$

With suitable values of $\beta_{1}, \beta_{2}, \ldots, \beta_{n}, \beta_{n+1}$ the observer can track the states so that $\widehat{x}_{1} \approx x_{1}, \widehat{x}_{2} \approx x_{2}, \ldots, \widehat{x}_{n} \approx x_{n}, \widehat{x}_{n+1} \approx$ $x_{n+1}=F(X, t)$.

Note that a third-order observer is required when implementing an ADR controller for a second-order system. With a good estimation of all states of the system and the additional state, the control signal in the ADR controller is defined as

$$
u(t)=-\widehat{x}_{n+1}+k_{1}(t) \widehat{e}_{1}+k_{2}(t) \widehat{e}_{2}+\cdots+k_{n}(t) \widehat{e}_{n}
$$

where $k_{1}(t), k_{2}(t), \ldots, k_{n}(t)$ are the time-varying gains of the controller. By substituting (7) into (5), the uncertain and 
disturbed system (1) can be reduced to a simple disturbancefree system represented as set of linear integrators:

$$
\begin{gathered}
\dot{x}_{1}=x_{2} \\
\dot{x}_{2}=x_{3} \\
\vdots \\
\dot{x}_{n}=k_{1} \widehat{e}_{1}+k_{2} \widehat{e}_{2}+\cdots+k_{n} \widehat{e}_{n}
\end{gathered}
$$

The objective in any control is to obtain an accurate estimation and then a cancellation of the total disturbance along with an accurate tracking of the reference. The speed at which the observer estimates the total disturbance $F(X, t)$ to be cancelled by the controller in real time is crucial. The procedure to determine the parameters of the ADR controller for the tracking control problem of the chaotic system considered in this study is explained in the next section.

\section{Parameter Optimization of ADR Controller}

4.1. Selecting Parameters. Parameter tuning in the ADR controller is usually a manual process. It is based on experience in the control field and is relatively difficult. Moreover, the numerous parameters requiring adjustments by manual methods weaken the control performance. The parameters of the ADR controller are both observer-related and controllerrelated. For the observer, one set of parameters that are required are the gains. With the parameterization technique proposed in [21], the roots of the characteristic polynomial

$$
\begin{aligned}
\lambda(s)= & s^{n+1}+\beta_{1} s^{n}+\beta_{2} s^{n-1}+\cdots+\beta_{n-1} s^{2}+\beta_{n} s \\
& +\beta_{n+1}
\end{aligned}
$$

are compared with polynomial

$$
G_{o}(s)=\left(s+\omega_{o}(t)\right)^{n+1}
$$

thence placing all of the observers poles at $-\omega_{0}$ and reducing the characteristic polynomial to Hurwitz-type. The same can be done to determine the controller gains $k_{1}, k_{2}, \ldots k_{n}$. The roots of the characteristic polynomial

$$
\lambda_{c}(s)=s^{n}+k_{n} s^{n-1}+\cdots+k_{2} s+k_{1}
$$

are compared with the following polynomial:

$$
G_{c}(s)=\left(s+\omega_{c}(t)\right)^{n}
$$

so that all the poles are placed at $-\omega_{c}$, where $\omega_{c}$ is the bandwidth of the feedback controller.

A higher bandwidth results in better reference signal tracking and disturbance rejection. Nevertheless, the sensor noise and dynamic uncertainties are two factors that limit the maximum closed-loop bandwidth [19].

If the initial value of the extended observer is different from the initial value of the plant, the phenomenon of peaking appears for very large $\omega_{0}$. This can affect significantly the convergence of the extended observer. A possible way to mitigate peaking is to let the observer and the controller bandwidth vary with time as, for example, [24]

$$
\boldsymbol{\omega}_{\boldsymbol{o}}(\boldsymbol{t})=A \frac{1-e^{-B t}}{1+e^{-B t}}
$$

where $A$ and $B$ are two positive constants. If $\omega_{c}(t)=\omega_{o}(t) / C$, with constant $C$ that can be chosen from the range [2-10], then, the parameters of the ADR controller, $A, B$, and $C$, can be optimized via an appropriate selection of an objective function.

4.2. Objective Function. The selection of the objective function is important for accuracy during parameter setting. To achieve good tracking performance, and at the same time an acceptable control signal, a fitness function $J$ is introduced that is a combination of the integral of the absolute error (IAE) and the integral of the absolute control signal (IAU). The IAE performance index is adopted to obtain stability and accuracy of the tracking process; the IAU is included to minimize the control signal so that the need to limit the control signal using saturation function is eliminated. In practical applications, there are limiting conditions on the control energy that leads to a failure to achieve the desired performance. The minimization problem is formulated as

$$
J(A, B, C)=\lambda \int_{0}^{t}\left|e_{1}(t)\right| d t+\int_{0}^{t}|u(t)| d t
$$

where $\lambda$ is a positive constant. Setting $\lambda$ large leads to a more accurate tracking and a larger control signal.

4.3. Optimization Method. Genetic algorithms (GAs) [22, 23] are optimization methodologies based on Darwinian principles of evolutionary biology. They are often used to solve nonlinear or nondifferentiable optimization problems. As shown in Figure 1, a basic genetic algorithm consists of the following steps:

1. Initial population creation: randomly generate $n$ chromosomes that are possible solutions of the problem.

(2) Calculating the fitness: by running the simulation obtain the fitness of the $\mathrm{n}$ chromosomes using the objective function.

(3) Generating the new population: by applying the GA operators until a new population is complete.

(4) Replacing: combine the old with newly generated solutions.

(5) Termination: if the termination criterion is satisfied, stop.

6. Loop: go to Step (2) for fitness evaluation.

The GA operators in step (3) are (a) Selection: based on their fitness, select two parent chromosomes, (b) Crossover: Cross over the parents to form new children, (c) Mutation: mutate new offspring at a randomly selected position in the chromosome, and (d) Acceptance: place new children in the 


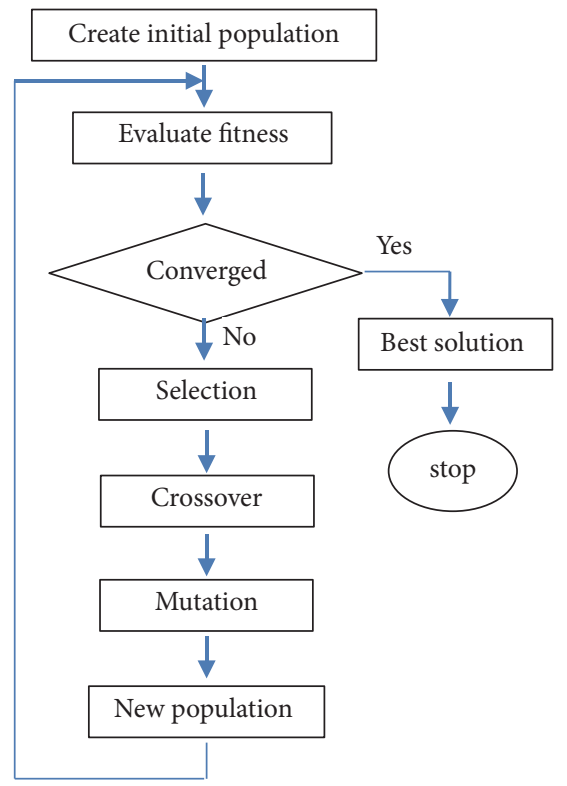

FIGURE 1: Basic genetic algorithm.

new population. Selection methods include, for example, the roulette-wheel selection, random selection, and tournament selection. The crossover operation can be single-point, twopoint, or uniform. Mutation prevents the algorithm from being stuck in a local minimum. The mutation can be performed using procedures such as Flipping, Interchanging, and Reversing. The algorithm is terminated when the population converges through a variety of convergence criteria. A couple of popular ones are the fixed number of generations, and the best objective value is no longer changing.

\section{Simulation Results}

In this section, two well-known chaotic systems, the Duffing and Genesio-Tesi systems, are considered to demonstrate by numerical experiments the effectiveness of the proposed ADR control scheme.

5.1. Duffing System (DS). A nonlinear oscillator with a cubic stiffness term to describe the hardening spring effect observed in many mechanical problems was introduced by Duffing. Duffing's equation has the form

$$
\ddot{x}+a_{1} \dot{x}+a_{2} x+a_{3} x^{3}=u(t)+q \cos \omega t
$$

where $x$ is the oscillation displacement, $a_{1}$ the damping constant, $a_{2}$ the linear stiffness constant, $a_{3}$ the cubic stiffness constant, $q$ the excitation amplitude, and $\omega$ the excitation frequency. To establish the dynamic behavior of the chaotic DS, we select parameter settings: $a_{1}=-1.1, a_{2}=0.4, a_{3}=$ $-1, q=1.3$, and $\omega=1.8 \mathrm{rad} / \mathrm{s}$. From the DS response for the initial conditions $x=1, \dot{x}=2$ (Figure 2), the system develops chaotic behavior when no control signal is applied.
To test the performance of the ADR controller in its tracking task, the DS is controlled to follow a reference signal and its derivative given by

$$
\begin{aligned}
& r_{1}(t)=10 \sin (t) \\
& r_{2}(t)=10 \cos (t)
\end{aligned}
$$

By defining the states of (15), $x_{1}=x$ and $x_{2}=\dot{x}$, it can be rewritten as two first-order ordinary differential equations,

$$
\begin{aligned}
\dot{x}_{1}(t)= & x_{2}(t) \\
\dot{x}_{2}(t)= & a_{1} x_{1}(t)+a_{2} x_{2}(t)+a_{3} x_{1}^{3}(t)+q \cos (\omega t) \\
& +u(t) \\
y(t)= & x_{1}(t)
\end{aligned}
$$

Taking $F\left(x_{1}, x_{2}, \omega, t\right)=a_{1} x_{1}(t)+a_{2} x_{2}(t)+a_{3} x_{1}^{3}(t)+q \cos (\omega t)$ as the extended state $x_{3}$, (17) can be rewritten as

$$
\begin{aligned}
& \dot{x}_{1}=x_{2} \\
& \dot{x}_{2}=x_{3}+u(t) \\
& \dot{x}_{3}=\dot{F}\left(x_{1}, x_{2}, \omega, t\right)
\end{aligned}
$$

The ESO for a second-order system is as follows:

$$
\begin{aligned}
& \dot{\hat{x}}_{1}(t)=\widehat{x}_{2}(t)+3 \omega_{o}(t)\left(y(t)-\widehat{x}_{1}(t)\right) \\
& \dot{\hat{x}}_{2}(t)=\widehat{x}_{3}(t)+3 \omega_{o}^{2}(t)\left(y(t)-\widehat{x}_{1}(t)\right) \\
& \dot{\hat{x}}_{3}(t)=\omega_{o}^{3}(t)\left(y(t)-\widehat{x}_{1}(t)\right)
\end{aligned}
$$

The feedback controller is described by

$$
u(t)=-\widehat{x}_{3}+\omega_{c}^{2}(t) \widehat{e}_{1}+2 \omega_{c} \widehat{e}_{2}
$$



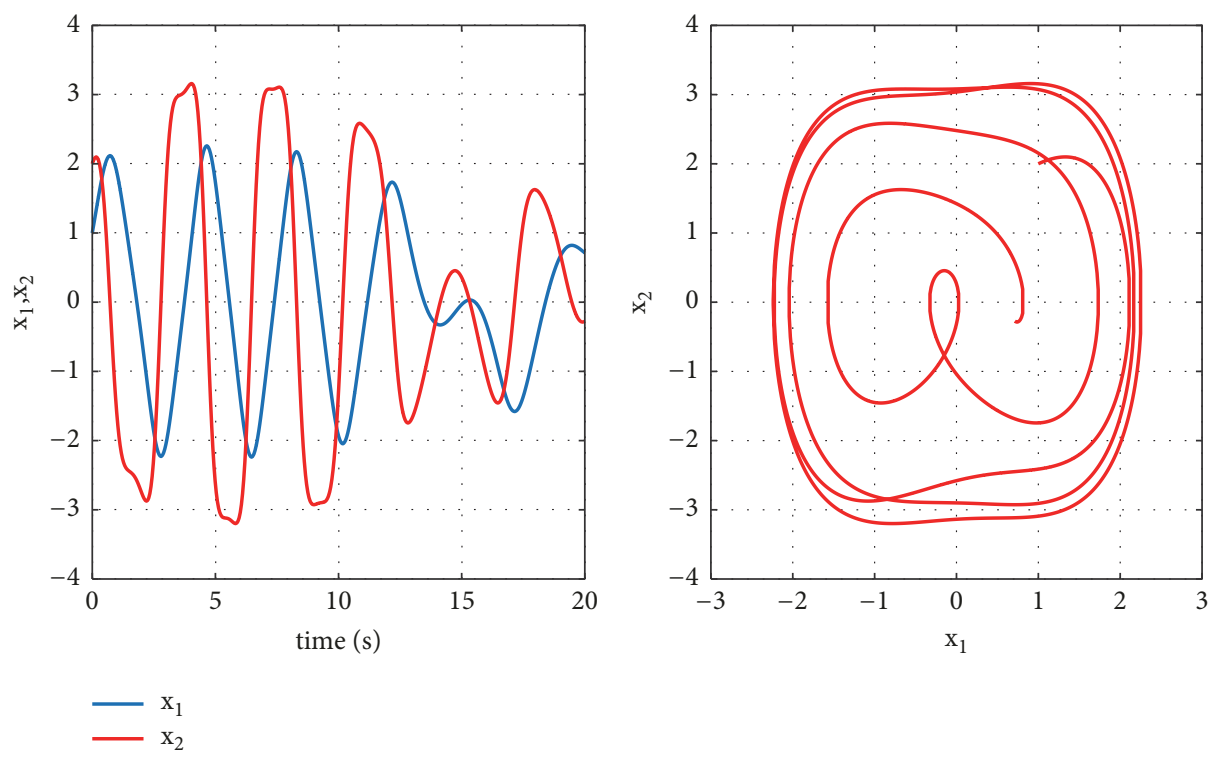

FIGURE 2: Chaotic behavior of DS without the control input in 20 seconds.
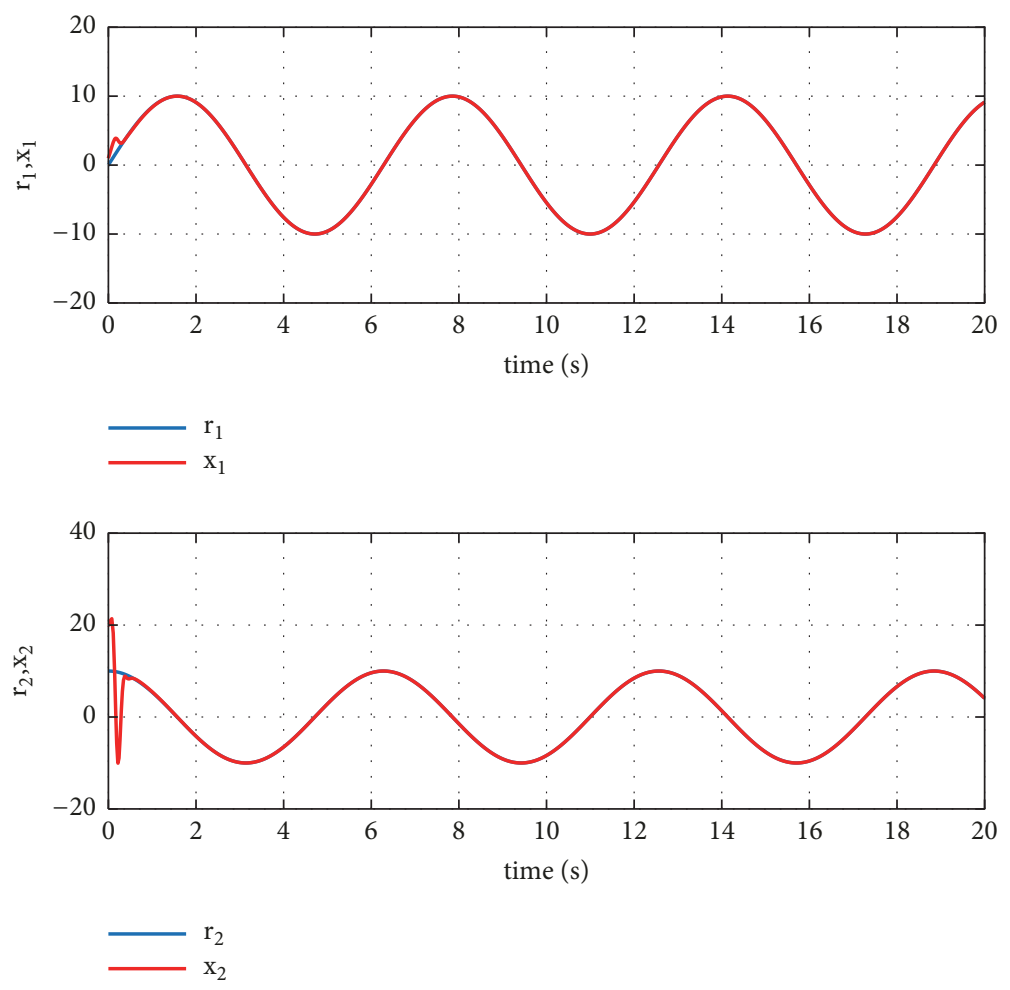

FIgURE 3: Controlled time response of the states $x_{1}, x_{2}$.

To optimize the three parameters $A, B$, and $C$, the lower and upper bounds of the parameters are selected as (after a number of experimental simulations) $A=[5,200], B=$ $[0.1,1]$, and $C=[2,10]$. The parameter settings of the GA are as follows: (i) a population size of 20-the tournament selection is applied to all chromosomes as the number of chromosomes in the population is small; (ii) a two-point crossover function and a uniform mutation function; (iii) during the optimization process, the stopping condition is invoked if the maximum number of generations reaches 100; and (iv) to increase the accuracy of tracking, a value of $\lambda=$ 100 is set in objective function.

The optimized controller parameters are then $A=$ $170.0221, B=0.9985$, and $C=1.300$. The simulation results are presented in Figures 3-6. The time-dependent responses of the states of the DS (Figure 2) show the states exactly 


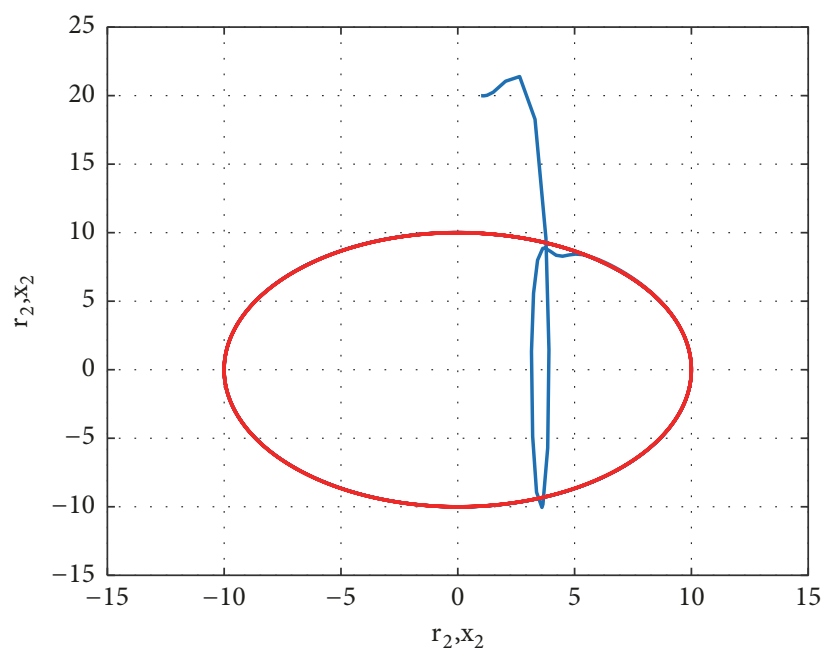

FIGURE 4: The controlled trajectory of the DS system.
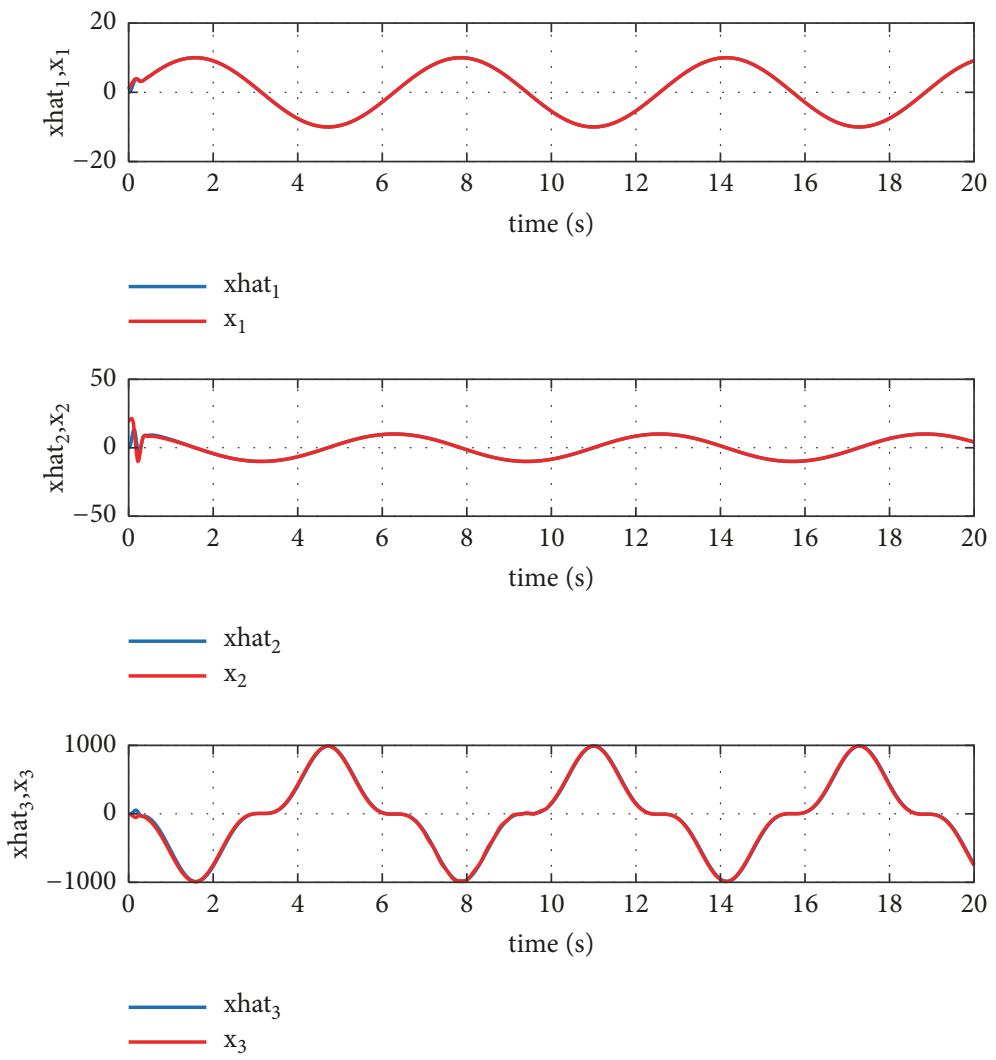

FIGURE 5: The performance of the ESO in estimating the states.

following the reference signal and its derivative after a short time. A good view of the tracking of the DS of the reference signal is seen in the phase-plane plot (Figure 4). The ESO performs well in estimating the two states of the system and the extended state in less than one second (Figure 5). The control effort required to force the DS follows the reference signal (Figure 6); note that the control signal required to control the DS is limited because the optimization of the ADR controller parameters based on the objective function (14) includes the minimization of the control signal as part of it. Therefore, the need to saturate the control signal is eliminated. The ADR controller parameter settings obtained from the minimization process leads to a very fast control of the system's chaotic behavior.

5.2. Genesio-Tesi System (GST). As our second example, the GTS is considered having the form,

$$
\begin{aligned}
& \dot{x}_{1}=x_{2} \\
& \dot{x}_{2}=x_{3}
\end{aligned}
$$




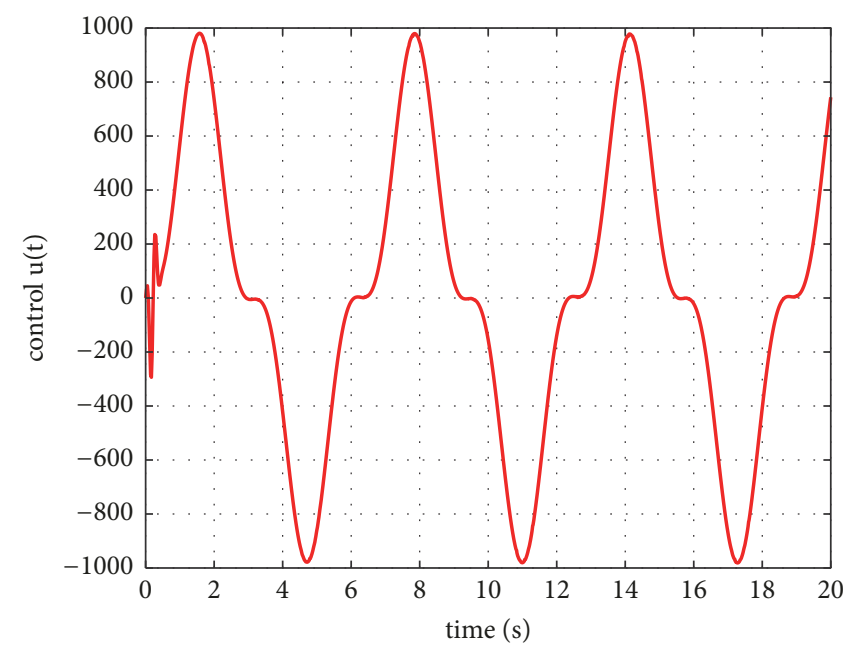

FIgURE 6: The time response of the control signal $u(t)$.
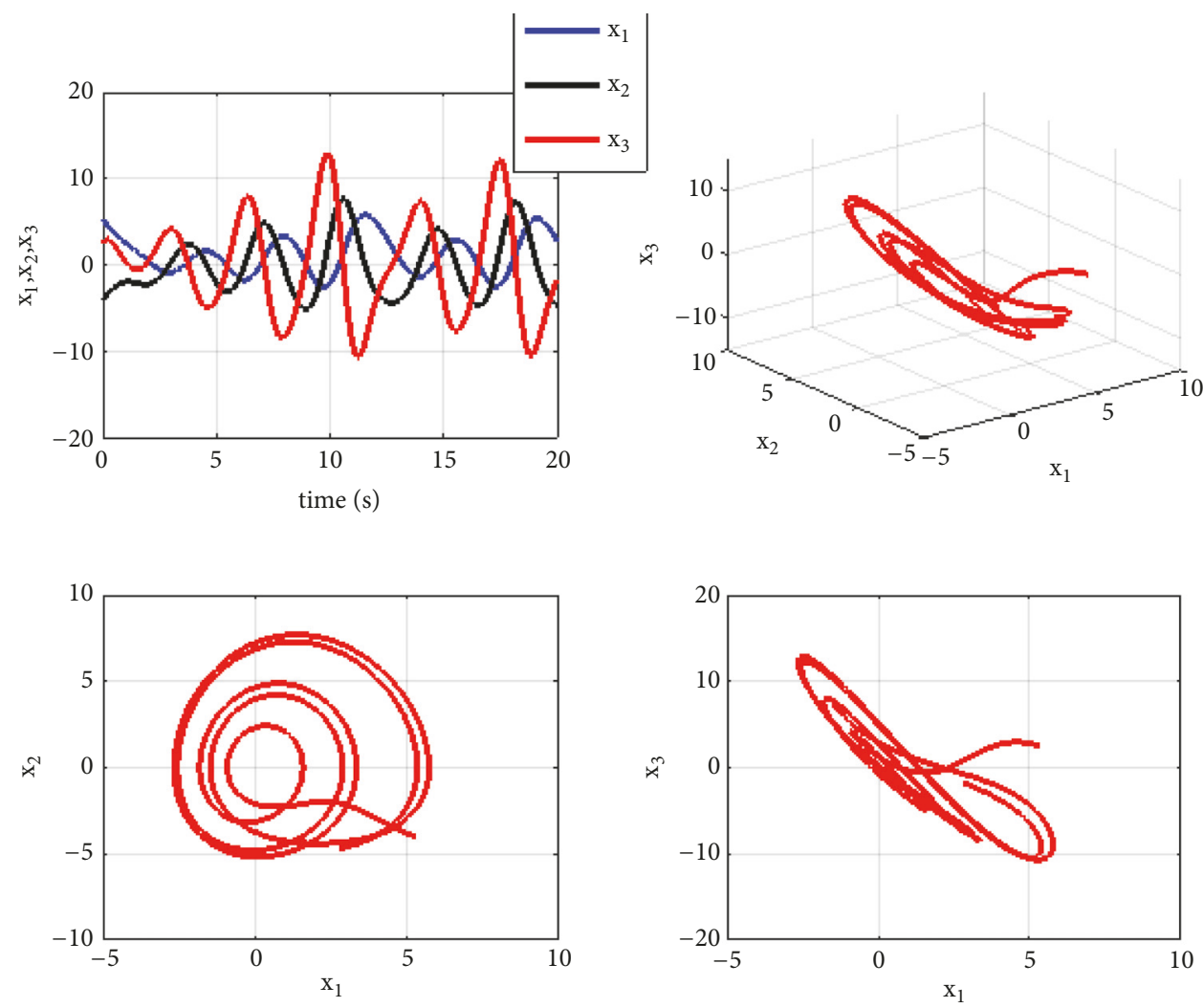

FIgURE 7: Chaotic trajectories of Genesio-Tesi system (GTS).

$$
\begin{aligned}
\dot{x}_{3}= & -a x_{1}-b x_{2}-c x^{3}+m x_{1}^{2}+\Delta f\left(x_{1}, x_{2}, x_{3}, t\right) \\
& +d(t)+u(t)
\end{aligned}
$$

where $x_{1}, x_{2}$, and $x_{3}$ are the system states with $\left[x_{1}, x_{2}, x_{3}\right]^{T}$ the full-state vector, $a, b, c$, and $m$ are unknown positive real constants satisfying $a b<c, \Delta f\left(x_{1}, x_{2}, x_{3}, t\right)$ is an unknown function depending on the full state and time, $u(t)$ is the control signal to be designed with control input $u(t)=0$, $\triangle f\left(x_{1}, x_{2}, x_{3}, t\right)=0$, and $d(t)=0$. The chaotic response of the GTS (Figure 7) was established using the initial conditions $\left[x_{10}=5.3, x_{20}=-4, x_{30}=2.5\right]$ for the variables and $\mathrm{a}$ running time of 20 seconds.

To test the performance of the ADRC in the task of tracking, the GTS is controlled to follow the reference signal and its derivatives given by 

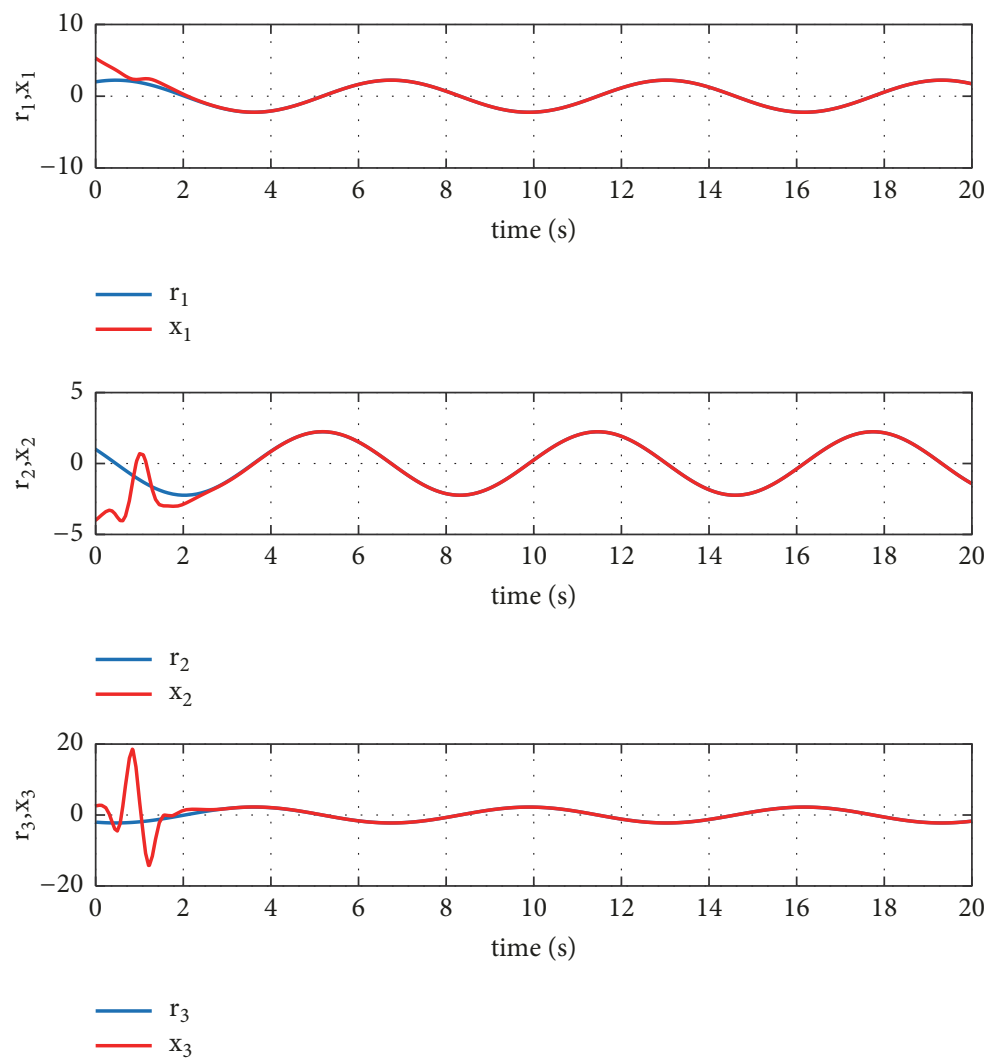

Figure 8: Time responses of the states.

$$
\begin{aligned}
& r_{1}(t)=2 \cos (t)+\sin (t) \\
& r_{2}(t)=\cos (t)-2 \sin (t) \\
& r_{3}(t)=-2 \cos (t)-\sin (t)
\end{aligned}
$$

The uncertain term added is

$$
\begin{aligned}
\Delta f & \left(x_{1}, x_{2}, x_{3}, t\right) \\
& =0.05 \sin \left(\pi x_{1}\right) \sin \left(2 \pi x_{2}\right) \sin \left(3 \pi x_{3}\right)
\end{aligned}
$$

and the external disturbance is

$$
d(t)=0.002 \cos (t)
$$

To estimate the three states and the total uncertainty, the following fourth-order ESO is required:

$$
\begin{aligned}
& \dot{\hat{x}}_{1}(t)=\widehat{x}_{2}(t)+4_{1} \omega_{o}(t)\left(x_{1}(t)-\widehat{x}_{1}(t)\right) \\
& \dot{\hat{x}}_{2}(t)=\widehat{x}_{3}(t)+6 \omega_{o}^{2}(t)\left(x_{1}(t)-\widehat{x}_{1}(t)\right) \\
& \dot{\hat{x}}_{3}(t)=\widehat{x}_{4}(t)+4 \omega_{o}^{3}(t)\left(x_{1}(t)-\widehat{x}_{1}(t)\right)+u(t) \\
& \dot{\hat{x}}_{4}(t)=\alpha_{4} \omega_{o}^{4}(t)\left(x_{1}(t)-\widehat{x}_{1}(t)\right)
\end{aligned}
$$

The feedback controller is described by

$$
u(t)=-\widehat{x}_{4}+\omega_{c}^{3}(t) \widehat{e}_{1}+3 \omega_{c}^{2}(t) \widehat{e}_{2}+3 \omega_{c}(t) \widehat{e}_{3}
$$

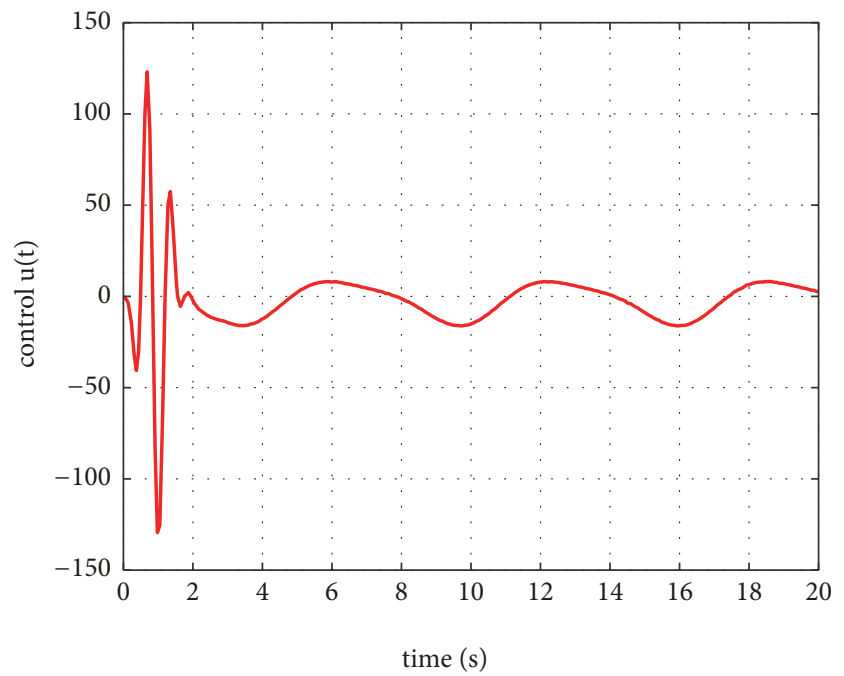

FIgURE 9: Time response of control input $(\mathrm{u}(\mathrm{t}))$.

Using GA optimization, the control parameter settings obtained are $A=24.960, B=0.535, C=1.985$ using the same GA parameters and options adopted in the previous section. The simulation results are presented in Figures 8-12. The time-dependent responses of the states of the GTS (Figure 8) show again that the states exactly follow the reference signal and its derivative after a short time. Figure 9 shows the control 

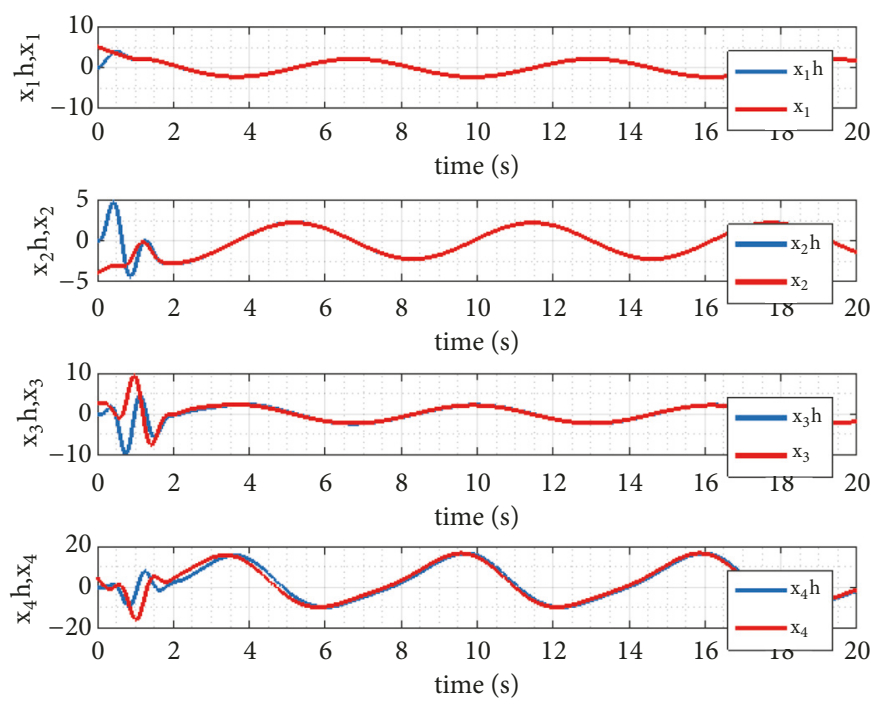

Figure 10: The performance of the ESO in estimating the states.

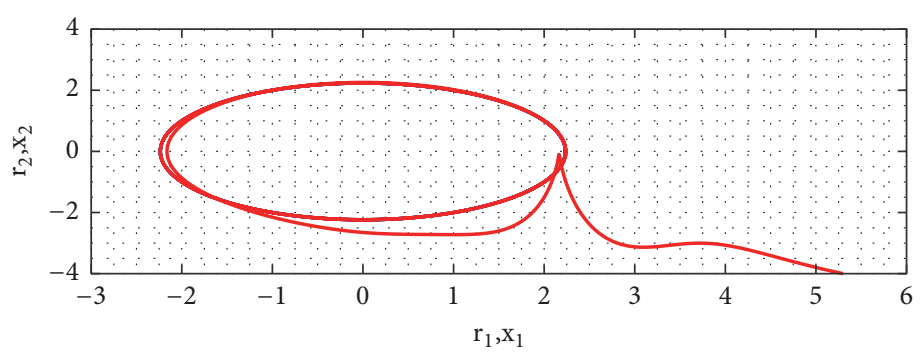

- Reference

Response

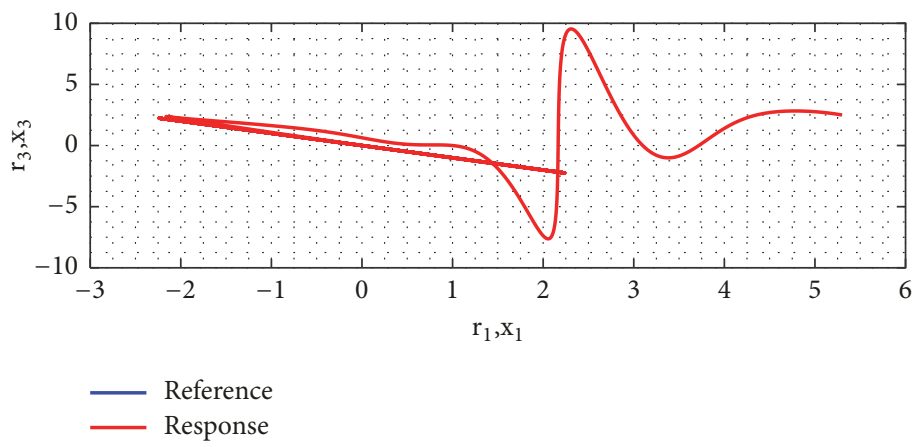

FIgURE 11: The controlled trajectories of the GT system.

effort required to follow the reference signal. The ESO also performs well in estimating the three states of the system and the extended state (Figure 10). Figure 11 shows the controlled trajectories of GTS in the $x_{1} x_{2}$ and $x_{1} x_{3}$ planes. Figure 12 shows the time-varying observer and controller bandwidths.

\section{Conclusion}

An ADR controller has been developed to control chaotic systems and applied in two well-known examples to demonstrate its capabilities. The merit of this approach is the use of time-varying parameters for the observer as well as for the feedback controller. The results obtained in simulations clearly show the controller performs well in controlling both chaotic systems with bounded uncertainty and disturbance to any arbitrarily desired reference signal with high accuracy. The results also show that the ADR method holds promise in providing solutions in controlling chaos. The ADR has been applied to control a second-order DS and a third-order GTS. A suitable selection of the control parameter settings was observed to influence the control effort and error. Hence, the genetic algorithm was applied to obtain optimal parameter 


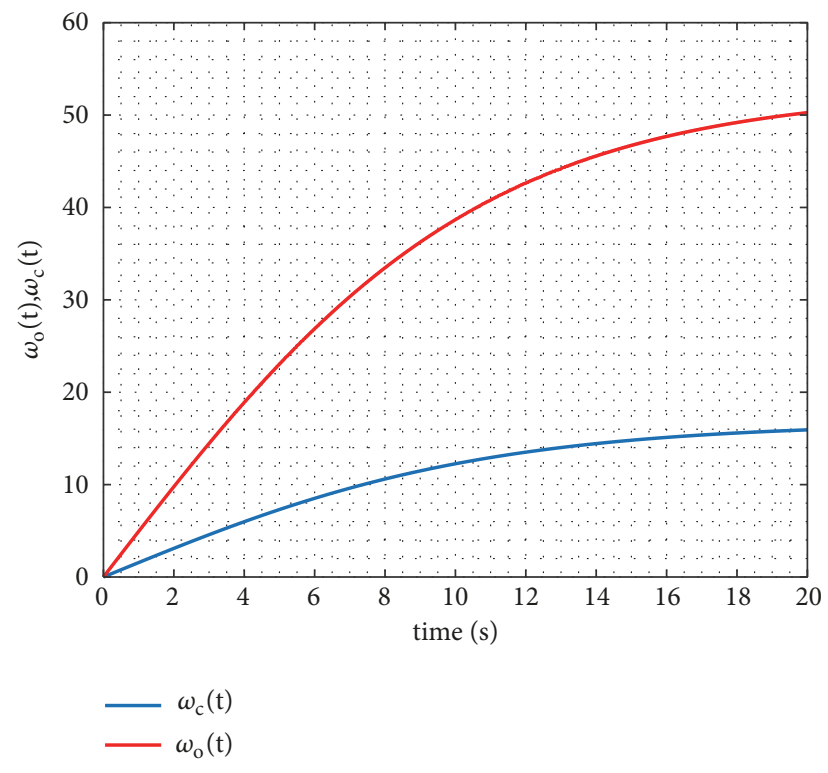

FIgURE 12: The time-varying observer and controller bandwidths.

settings of the ADR controller. In further study, the ADR controller is to be applied to the synchronization of chaos.

\section{Data Availability}

The data used to support the findings of this study are available from the corresponding author upon request.

\section{Conflicts of Interest}

The author declares that there are no conflicts of interest.

\section{References}

[1] L. M. Pecora, T. L. Carroll, G. A. Johnson, D. J. Mar, and J. F. Heagy, "Fundamentals of synchronization in chaotic systems, concepts, and applications," Chaos: An Interdisciplinary Journal of Nonlinear Science, vol. 7, no. 4, pp. 520-543, 1997.

[2] X. Zhang, X. Liu, and Q. Zhu, "Adaptive chatter free sliding mode control for a class of uncertain chaotic systems," Applied Mathematics and Computation, vol. 232, pp. 431-435, 2014.

[3] B. Wang and S. Zhong, "Observer-based control on a chaotic system with unknowns and uncertainties," Optik - International Journal for Light and Electron Optics, vol. 137, pp. 167-174, 2017.

[4] R. Luo and H. Su, "Finite-time control and synchronization of a class of systems via the twisting controller," Chinese Journal of Physics, vol. 55, no. 6, pp. 2199-2207, 2017.

[5] S. Dadras, H. R. Momeni, and V. J. Majd, "Sliding mode control for uncertain new chaotic dynamical system," Chaos, Solitons \& Fractals, vol. 41, no. 4, pp. 1857-1862, 2009.

[6] J. Sun, Y. Wang, Y. Wang, and Y. Shen, "Finite-time synchronization between two complex-variable chaotic systems with unknown parameters via nonsingular terminal sliding mode control," Nonlinear Dynamics, vol. 85, no. 2, pp. 1105-1117, 2016.

[7] X. Su, X. Liu, P. Shi, and R. Yang, "Sliding Mode Control of Discrete-Time Switched Systems with Repeated Scalar Nonlinearities," IEEE Transactions on Automatic Control, vol. 62, no. 9, pp. 4604-4610, 2017.
[8] J. Sun, Y. Wu, G. Cui, and Y. Wang, "Finite-time real combination synchronization of three complex-variable chaotic systems with unknown parameters via sliding mode control," Nonlinear Dynamics, vol. 88, no. 3, pp. 1677-1690, 2017.

[9] K.-M. Chang, "Adaptive control for a class of chaotic systems with nonlinear inputs and disturbances," Chaos, Solitons \& Fractals, vol. 36, no. 2, pp. 460-468, 2008.

[10] J. Wen and C.-S. Jiang, "Adaptive fuzzy control for a class of chaotic systems with nonaffine inputs," Communications in Nonlinear Science and Numerical Simulation, vol. 16, no. 1, pp. 475-492, 2011.

[11] C. Hua, X. Guan, X. Li, and P. Shi, "Adaptive observer-based control for a class of chaotic systems," Chaos, Solitons \& Fractals, vol. 22, no. 1, pp. 103-110, 2004.

[12] J. Zhou and M. J. Er, "Adaptive output control of a class of uncertain chaotic systems," Systems \& Control Letters, vol. 56, no. 6, pp. 452-460, 2007.

[13] N. Jia and T. Wang, "Chaos control and hybrid projective synchronization for a class of new chaotic systems," Computers \& Mathematics with Applications. An International Journal, vol. 62, no. 12, pp. 4783-4795, 2011.

[14] S. Dadras and H. R. Momeni, "Control uncertain GenesioTesi chaotic system: Adaptive sliding mode approach," Chaos, Solitons \& Fractals, vol. 42, no. 5, pp. 3140-3146, 2009.

[15] D. Zhang, J. Mei, and P. Miao, "Global finite-time synchronization of different dimensional chaotic systems," Applied Mathematical Modelling: Simulation and Computation for Engineering and Environmental Systems, vol. 48, pp. 303-315, 2017.

[16] H. Handa and B. B. Sharma, "Novel adaptive feedback synchronization scheme for a class of chaotic systems with and without parametric uncertainty," Chaos, Solitons \& Fractals, vol. 86, pp. 50-63, 2016.

[17] Jin Xing and Fangfang Zhang, "An Approach of Tracking Control for Chaotic Systems," Journal of Control Science and Engineering, vol. 2016, Article ID 9735264, 8 pages, 2016.

[18] Y.-C. Chang, "A robust tracking control for a class of uncertain chaotic systems," Asian Journal of Control, vol. 15, no. 6, pp. 1752-1763, 2013.

[19] J. Yu, J. Lei, and L. Wang, "Backstepping synchronization of chaotic system based on equivalent transfer function method," Optik - International Journal for Light and Electron Optics, vol. 130, pp. 900-913, 2017.

[20] J. Lei and L. Wang, "Backstepping synchronous control of chaotic system with reduced number of active inputs," Optik International Journal for Light and Electron Optics, vol. 127, no. 23, pp. 11364-11373, 2016.

[21] J. Q. Han, "From PID to active disturbance rejection control," IEEE Transactions on Industrial Electronics, vol. 56, no. 3, pp. 900-906, 2009.

[22] Z. Gao, "Scaling and bandwidth-parameterization based controller tuning," in Proceedings of the American Control Conference, pp. 4989-4996, Denver, Colo, USA, June 2003.

[23] Q. Zheng and Z. Gao, "Predictive active disturbance rejection control for processes with time delay," ISA Transactions ${ }^{\circledR}$, vol. 53, no. 4, pp. 873-881, 2014.

[24] J. Liu, Advanced Sliding Mode Control for Mechanical Systems: Design, Analysis and MATLAB Simulation: with 165 Figures, Tsinghua University Press, Beijing, China, 2012. 


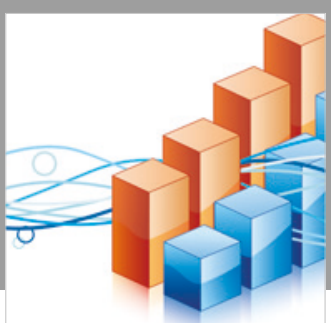

Advances in

Operations Research

\section{-n-m}
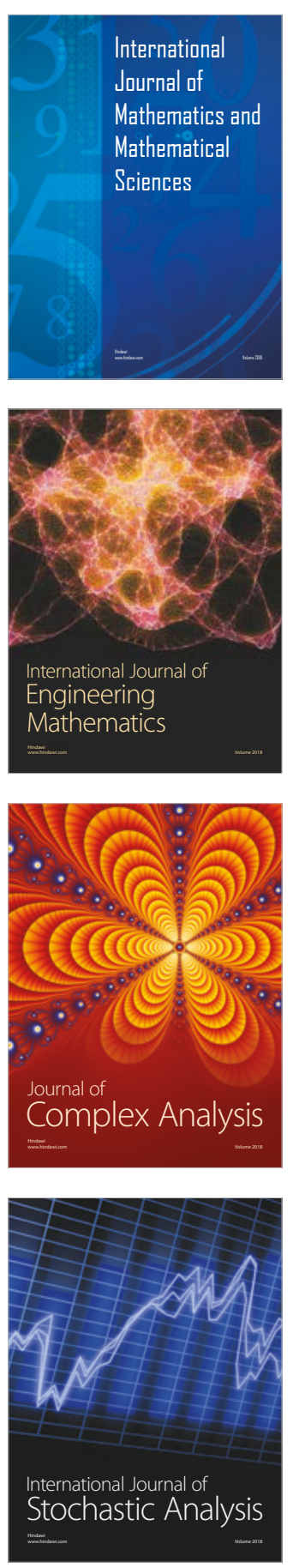
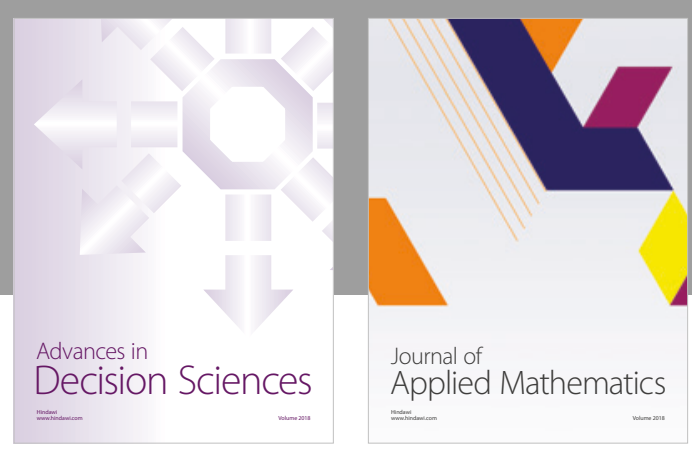

Journal of

Applied Mathematics
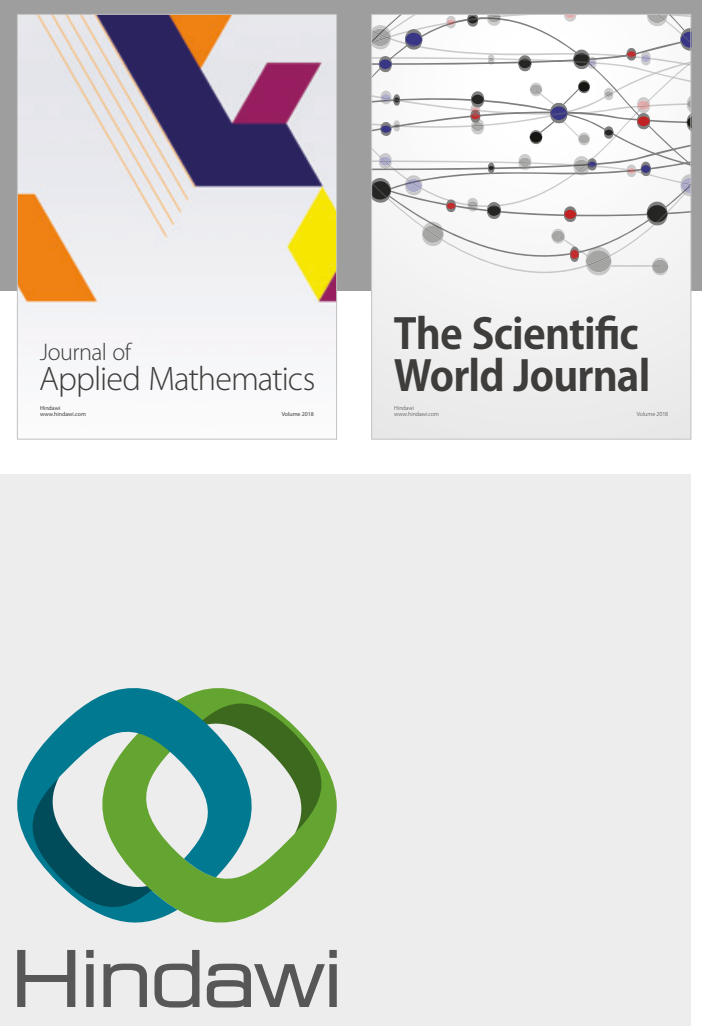

Submit your manuscripts at

www.hindawi.com

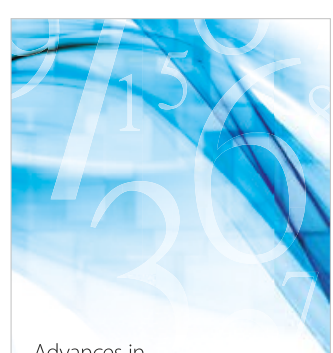

Advances in
Numerical Analysis
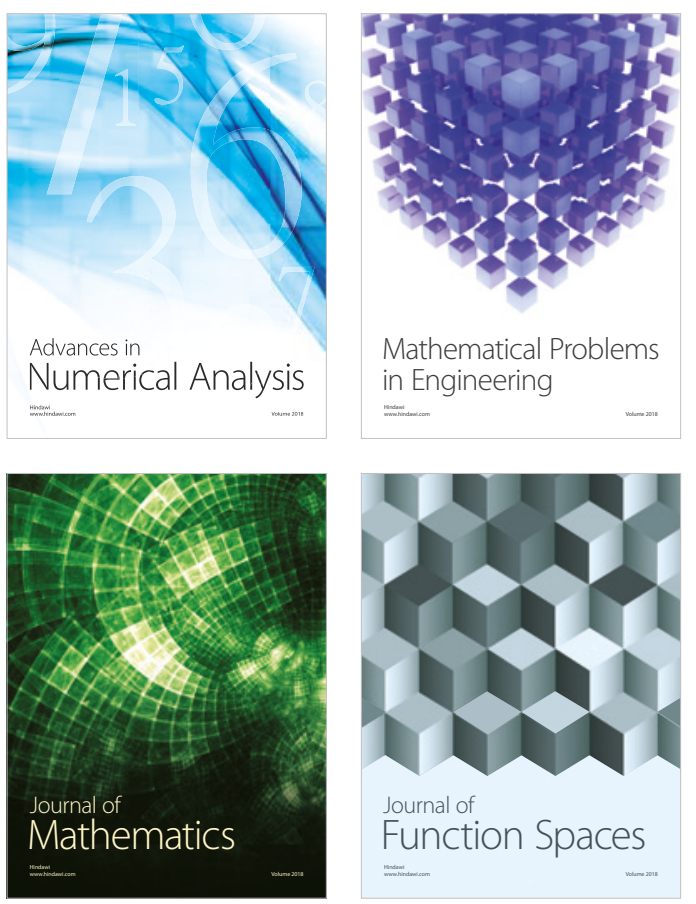

Mathematical Problems in Engineering

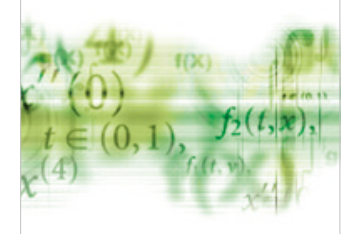

International Journal of

Differential Equations

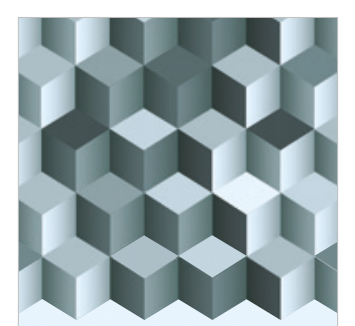

Journal of

Function Spaces

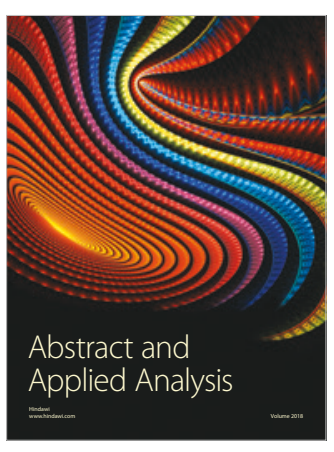

The Scientific

World Journal

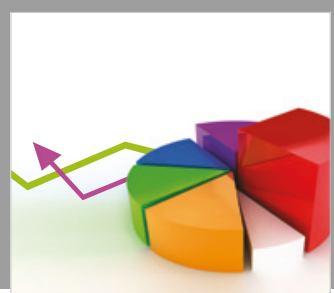

Journal of

Probability and Statistics
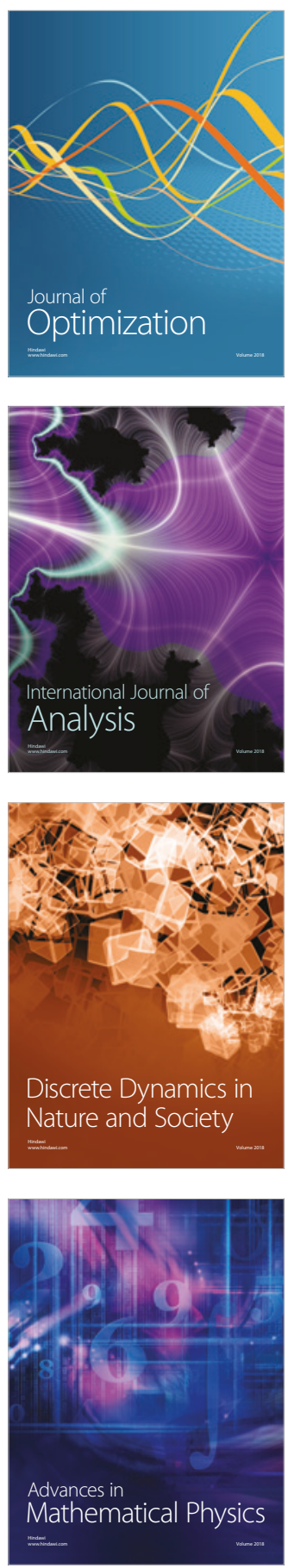